\title{
The sub-supersolution method for Kirchhoff systems: applications
}

\author{
Giovany M. Figueiredo ${ }^{1}$ and Antonio SuÁrez ${ }^{2}$,
}

1. Universidade Federal do Pará, Faculdade de Matemática

CEP: 66075-110 Belém - PA , Brazil

2. Dpto. de Ecuaciones Diferenciales y Análisis Numérico

Fac. de Matemáticas, Univ. de Sevilla

C/. Tarfia s/n, 41012 - Sevilla, Spain.

E-mail addresses: giovany@ufpa.br, suarez@us.es

ABSTRACT. In this paper we prove that the sub-supersolution method works for general Kirchhoff systems. We apply the cited method to prove the existence of positive solutions for some specific models.

AMS Subject Classification 2000. 35J35, 35J65,49J40.

KEYWORDS. Kirchhoff systems, Sub-Supersolutions method.

\section{Introduction}

In this note we study the existence of solutions of a nonlinear Kirchhoff system

$$
\begin{cases}-M_{1}\left(\left\|u_{1}\right\|^{2}\right) \Delta u_{1}=f_{1}\left(x, u_{1}, u_{2}\right) & \text { in } \Omega, \\ -M_{2}\left(\left\|u_{2}\right\|^{2}\right) \Delta u_{2}=f_{2}\left(x, u_{1}, u_{2}\right) & \text { in } \Omega, \\ u_{1}=u_{2}=0 & \text { on } \partial \Omega\end{cases}
$$

where $\Omega \subset \mathbb{R}^{N}, N \geq 1$, is a regular and bounded domain,

$$
\|u\|^{2}:=\int_{\Omega}|\nabla u|^{2} d x, \quad \text { for } u \in H_{0}^{1}(\Omega)
$$

$M_{i}, i=1,2$ are continuous functions verifying

$$
M_{i}: \mathbb{R}_{+} \mapsto \mathbb{R}_{+} \quad \text { and } \exists m_{0}>0 \text { such that } M_{i}(t) \geq m_{0}>0 \forall t \in \mathbb{R}_{+},
$$

and $f_{i} \in C\left(\bar{\Omega} \times \mathbb{R}^{2}\right)$. We assume $(M)$ along the paper. 
Basically, in our knowledge, similar systems to (1.1) have been analyzed in several papers. In [8], [3], [4], [6], [10] and references therein, variational methods have been applied to prove existence and multiplicity of positive solutions for systems as (1.1). In [1] and [2] the sub-supersolution method has been used to prove the existence of solution with $M_{i}$ increasing and bounded from above and below for positive constants, that is, there exist positive constants $0<m_{i} \leq m_{i}^{\infty}<\infty$ such that

$$
0<m_{i} \leq M_{i}(t) \leq m_{i}^{\infty}<\infty \quad i=1,2, \quad \forall t \geq 0 .
$$

However, in both papers the authors use a comparison principle (see for instance Lemma 2.1 in [1]) which seems not to be correct, see [5].

In this paper, we prove that the sub-supersolution method works for system (1.1), when the sub-supersolution is defined in an appropriate way, see Theorem 3.3. Indeed, in this case, the definition of sub-supersolution depends on the monotony of the non-linear reaction term (in a similar way to the local problems, see for instance [9]) and on the functions $M_{i}$. In order to prove this result, we transform our Kirchhoff system (1.1) into another with general non-local term depending only on the unknown variable $u_{i}$ but not the $\left\|u_{i}\right\|^{2}$. So, as consequence, we establish a very general sub-supersolution method for for a large class of systems with nonlinear and non-local terms (see Theorem 2.2).

The paper is organized as follows. In Section 2 we show that the sub-supersolution method works for general non-local systems. In Section 3, under very general conditions on $M_{i}$, we transform our system (1.1) into a non-local systems, and apply the method of Section 2. Section 4 is devoted to apply our method for different particular systems.

\section{The sub-super method for non-local systems}

First of all we show that the sub-supersolution method works well for non-local systems of the following type

$$
\begin{cases}-\Delta u_{1}=g_{1}\left(x, u_{1}, u_{2}, B_{1}\left(u_{1}\right), B_{2}\left(u_{2}\right), C_{1}\left(u_{1}, u_{2}\right)\right) & \text { in } \Omega \\ -\Delta u_{2}=g_{2}\left(x, u_{1}, u_{2}, B_{1}\left(u_{1}\right), B_{2}\left(u_{2}\right), C_{2}\left(u_{1}, u_{2}\right)\right) & \text { in } \Omega \\ u_{1}=u_{2}=0 & \text { on } \partial \Omega\end{cases}
$$

where $g_{i}: \Omega \times \mathbb{R}^{5} \mapsto \mathbb{R}$ is a continuous function, $B_{i}: L^{\infty}(\Omega) \mapsto \mathbb{R}, C_{i}:\left(L^{\infty}(\Omega)\right)^{2} \mapsto \mathbb{R}$ are continuous operators. Given $w \leq z$ a.e. in $\Omega$, we denote by

$$
[w, z]:=\{u: w(x) \leq u(x) \leq z(x) \text { a.e. } x \in \Omega\} .
$$

Definition 2.1. We say that the pair $\left(\underline{u}_{1}, \bar{u}_{1}\right),\left(\underline{u}_{2}, \bar{u}_{2}\right)$, with $\underline{u}_{i}, \bar{u}_{i} \in H^{1}(\Omega) \cap L^{\infty}(\Omega)$, is a pair of sub-supersolution of (2.1) if

1. $\underline{u}_{i} \leq \bar{u}_{i}$ in $\Omega$ and $\underline{u}_{i} \leq 0 \leq \bar{u}_{i}$ on $\partial \Omega$ for $i=1,2$,

2.

$$
-\Delta \underline{u}_{1}-g_{1}\left(x, \underline{u}_{1}, v, B_{1}(u), B_{2}(v), C_{1}(u, v)\right) \leq 0 \leq-\Delta \bar{u}_{1}-g_{1}\left(x, \bar{u}_{1}, v, B_{1}(u), B_{2}(v), C_{1}(u, v)\right)
$$

in the weak sense for all $(u, v) \in\left[\underline{u}_{1}, \bar{u}_{1}\right] \times\left[\underline{u}_{2}, \bar{u}_{2}\right]$. 
3.

$$
-\Delta \underline{u}_{2}-g_{2}\left(x, u, \underline{u}_{2}, B_{1}(u), B_{2}(v), C_{2}(u, v)\right) \leq 0 \leq-\Delta \bar{u}_{2}-g_{2}\left(x, u, \bar{u}_{2}, B_{1}(u), B_{2}(v), C_{2}(u, v)\right)
$$

in the weak sense for all $(u, v) \in\left[\underline{u}_{1}, \bar{u}_{1}\right] \times\left[\underline{u}_{2}, \bar{u}_{2}\right]$.

The main result in this section is:

Theorem 2.2. Assume that there exists a pair of sub-supersolution of (2.1) in the sense of Definition 2.1. Then, there exists a solution $\left(u_{1}, u_{2}\right) \in\left(H_{0}^{1}(\Omega) \cap L^{\infty}(\Omega)\right)^{2}$ of (2.1) such that $u_{i} \in\left[\underline{u}_{i}, \bar{u}_{i}\right], i=1,2$.

Proof. For $i=1,2$, define the truncation operators

$$
T_{i} u(x):= \begin{cases}\bar{u}_{i}(x) & \text { if } u(x) \geq \bar{u}_{i}(x), \\ u(x) & \text { if } \underline{u}_{i}(x) \leq u(x) \leq \bar{u}_{i}(x), \\ \underline{u}_{i}(x) & \text { if } u(x) \leq \underline{u}_{i}(x),\end{cases}
$$

and the Nemytskii operators $F_{i}:\left(L^{\infty}(\Omega)\right)^{2} \mapsto L^{\infty}(\Omega)$ given by

$$
F_{i}\left(u_{1}, u_{2}\right)(x):=g_{i}\left(x, T_{1}\left(u_{1}\right)(x), T_{2}\left(u_{2}\right)(x), B_{1}\left(T_{1}\left(u_{1}\right)\right), B_{2}\left(T_{2}\left(u_{2}\right)\right), C_{i}\left(T_{1}\left(u_{1}\right), T_{2}\left(u_{2}\right)\right)\right) .
$$

It is clear that $F_{i}$ is continuous and bounded, because there exists $M>0$ such that

$$
\left\|F_{i}\left(u_{1}, u_{2}\right)\right\|_{\infty} \leq M \quad \text { for all } u_{1}, u_{2} \in L^{\infty}(\Omega) .
$$

Consider the problem

$$
\begin{cases}-\Delta w_{1}=F_{1}\left(u_{1}, u_{2}\right) & \text { in } \Omega, \\ -\Delta w_{2}=F_{2}\left(u_{1}, u_{2}\right) & \text { in } \Omega, \\ w_{1}=w_{2}=0 & \text { on } \partial \Omega .\end{cases}
$$

We can define the operator $\mathcal{T}$ by $\left(u_{1}, u_{2}\right) \mapsto\left(w_{1}, w_{2}\right):=\mathcal{T}\left(u_{1}, u_{2}\right)$ being $\left(w_{1}, w_{2}\right)$ the unique solution of (2.3). It is clear that $\mathcal{T}$ is well-defined, it is a compact operator and $\mathcal{T}\left(B_{M}\right) \subset B_{M}$ for some $M>0$, where $B_{M}$ denotes the ball in $\left(L^{\infty}(\Omega)\right)^{2}$ centered in $(0,0)$ and radius $M$. Hence, by the Schauder Fixed Point Theorem there exists $\left(u_{1}, u_{2}\right) \in$ $\left(L^{\infty}(\Omega)\right)^{2}$ such that $\left(u_{1}, u_{2}\right)=\mathcal{T}\left(u_{1}, u_{2}\right)$, and then

$$
\begin{cases}-\Delta u_{1}=F_{1}\left(u_{1}, u_{2}\right) & \text { in } \Omega \\ -\Delta u_{2}=F_{2}\left(u_{1}, u_{2}\right) & \text { in } \Omega \\ u_{1}=u_{2}=0 & \text { on } \partial \Omega .\end{cases}
$$

Now, we show that $u_{i} \in\left[\underline{u}_{i}, \bar{u}_{i}\right]$, which implies that $\left(u_{1}, u_{2}\right)$ is solution of $(2.1)$. Let us show that

$$
u_{1} \leq \bar{u}_{1} \quad \text { in } \Omega \text {, }
$$

the other inequalities can be proved similarly. Indeed, in the definition of supersolution of $\bar{u}_{1}$ we can take $u=T_{1}\left(u_{1}\right), v=T_{2}\left(u_{2}\right)$ and then,

$$
-\Delta \bar{u}_{1} \geq g_{1}\left(x, \bar{u}_{1}, T_{2}\left(u_{2}\right), B_{1}\left(T_{1}\left(u_{1}\right)\right), B_{2}\left(T_{2}\left(u_{2}\right)\right), C_{1}\left(T_{1}\left(u_{1}\right), T_{2}\left(u_{2}\right)\right)\right),
$$


and so, denoting $z:=\bar{u}_{1}-u_{1}$ we get

$$
\begin{aligned}
-\Delta z & \geq g_{1}\left(x, \bar{u}_{1}, T_{2}\left(u_{2}\right), B_{1}\left(T_{1}\left(u_{1}\right)\right), B_{2}\left(T_{2}\left(u_{2}\right)\right), C_{1}\left(T_{1}\left(u_{1}\right), T_{2}\left(u_{2}\right)\right)\right)-F\left(u_{1}, u_{1}\right) \\
& =g_{1}\left(x, \bar{u}_{1}, T_{2}\left(u_{2}\right), B_{1}\left(T_{1}\left(u_{1}\right)\right), B_{2}\left(T_{2}\left(u_{2}\right)\right), C_{1}\left(T_{1}\left(u_{1}\right), T_{2}\left(u_{2}\right)\right)\right) \\
& -g_{1}\left(x, T_{1}\left(u_{1}\right)(x), T_{2}\left(u_{2}\right)(x), B_{1}\left(T_{1}\left(u_{1}\right)\right), B_{2}\left(T_{2}\left(u_{2}\right)\right), C_{1}\left(T_{1}\left(u_{1}\right), T_{2}\left(u_{2}\right)\right)\right) .
\end{aligned}
$$

Now, multiplying by $\left(\bar{u}_{1}-u_{1}\right)^{-}$we obtain

$$
\int_{\Omega}\left|\nabla\left(\bar{u}_{1}-u_{1}\right)^{-}\right|^{2} \leq 0
$$

whence we conclude the result.

\section{The sub-supersolution for Kirchhoff systems}

First, we are going to transform (1.1) into a nonlocal system as (2.1). Indeed, define

$$
N_{i}(t):=M_{i}(t) t
$$

and assume that $N_{i}$ is invertible, and so define

$$
G_{i}(t)=N_{i}^{-1}(t)
$$

Finally, define the non-local operators $\mathcal{R}_{i}:\left(L^{\infty}(\Omega)\right)^{2} \mapsto \mathbb{R}$ by

$$
\mathcal{R}_{i}\left(u_{1}, u_{2}\right)=M_{i}\left(G_{i}\left(\int_{\Omega} f_{i}\left(x, u_{1}, u_{2}\right) u_{i}\right)\right) .
$$

Lemma 3.1. Assume that

$$
N_{i}, i=1,2 \text { are invertible. }
$$

Then, (1.1) is equivalent to

$$
\begin{cases}-\Delta u_{1}=F_{1}\left(x, u_{1}, u_{2}, C_{1}\left(u_{1}, u_{2}\right)\right) & \text { in } \Omega \\ -\Delta u_{2}=F_{2}\left(x, u_{1}, u_{2}, C_{2}\left(u_{1}, u_{2}\right)\right) & \text { in } \Omega \\ u_{1}=u_{2}=0 & \text { on } \partial \Omega\end{cases}
$$

where

$$
C_{i}\left(u_{1}, u_{2}\right)=\mathcal{R}_{i}\left(u_{1}, u_{2}\right), \quad F_{i}\left(x, t_{1}, t_{2}, r\right)=\frac{f_{i}\left(x, t_{1}, t_{2}\right)}{r}, i=1,2 .
$$

Proof. Assume that $\left(u_{1}, u_{2}\right)$ is solution of (1.1). Multiplying (1.1) by $u_{i}$ and integrating, we get

$$
M_{i}\left(\left\|u_{i}\right\|^{2}\right)\left\|u_{i}\right\|^{2}=\int_{\Omega} f_{i}\left(x, u_{1}, u_{2}\right) u_{i}
$$

and then,

$$
\left\|u_{i}\right\|^{2}=G_{i}\left(\int_{\Omega} f_{i}\left(x, u_{1}, u_{2}\right) u_{i}\right) \Longrightarrow M_{i}\left(\left\|u_{i}\right\|^{2}\right)=\mathcal{R}_{i}\left(u_{1}, u_{2}\right)
$$


By $(M), \mathcal{R}_{i}\left(u_{1}, u_{2}\right) \geq m_{0}$ and then we can divide by $\mathcal{R}_{i}\left(u_{1}, u_{2}\right)$. Hence, we conclude that $\left(u_{1}, u_{2}\right)$ is solution of $(3.1)$.

Reciprocally, if $\left(u_{1}, u_{2}\right)$ is solution of $(3.1)$, then multiplying by $u_{i}$ we obtain

$$
\left\|u_{i}\right\|^{2}=\frac{\int_{\Omega} f_{i}\left(x, u_{1}, u_{2}\right) u_{i}}{\mathcal{R}_{i}\left(u_{1}, u_{2}\right)}=\frac{\int_{\Omega} f_{i}\left(x, u_{1}, u_{2}\right) u_{i}}{M_{i}\left(G_{i}\left(\int_{\Omega} f_{i}\left(x, u_{1}, u_{2}\right) u_{i}\right)\right.}=G_{i}\left(\int_{\Omega} f_{i}\left(x, u_{1}, u_{2}\right) u_{i}\right)
$$

where we have used that $N_{i} \circ G_{i}(t)=t$, that is $M_{i}\left(G_{i}(t)\right) G_{i}(t)=t$. Applying $M_{i}$ in that above equality we get

$$
M_{i}\left(\left\|u_{i}\right\|^{2}\right)=\mathcal{R}_{i}\left(u_{1}, u_{2}\right),
$$

and so $\left(u_{1}, u_{2}\right)$ is solution of (1.1). This completes the proof.

As consequence of this result and Theorem 2.2, we have the following results.

Definition 3.2. We say that the pair $\left(\underline{u}_{1}, \bar{u}_{1}\right),\left(\underline{u}_{2}, \bar{u}_{2}\right)$, with $\underline{u}_{i}, \bar{u}_{i} \in H^{1}(\Omega) \cap L^{\infty}(\Omega)$, is a pair of sub-supersolution of (1.1) if

1. $\underline{u}_{i} \leq \bar{u}_{i}$ in $\Omega$ and $\underline{u}_{i} \leq 0 \leq \bar{u}_{i}$ on $\partial \Omega$ for $i=1,2$,

2.

$$
-\mathcal{R}_{1}(u, v) \Delta \underline{u}_{1}-f_{1}\left(x, \underline{u}_{1}, v\right) \leq 0 \leq-\mathcal{R}_{1}(u, v) \Delta \bar{u}_{1}-f_{1}\left(x, \bar{u}_{1}, v\right)
$$

in the weak sense for all $(u, v) \in\left[\underline{u}_{1}, \bar{u}_{1}\right] \times\left[\underline{u}_{2}, \bar{u}_{2}\right]$.

3.

$$
-\mathcal{R}_{2}(u, v) \Delta \underline{u}_{2}-f_{2}\left(x, u, \underline{u}_{2}\right) \leq 0 \leq-\mathcal{R}_{2}(u, v) \Delta \bar{u}_{2}-f_{2}\left(x, u, \bar{u}_{2}\right)
$$

in the weak sense for all $(u, v) \in\left[\underline{u}_{1}, \bar{u}_{1}\right] \times\left[\underline{u}_{2}, \bar{u}_{2}\right]$.

Theorem 3.3. Assume (M) and (N). If there exists a pair of sub-supersolution of (3.1) in the sense of Definition 3.2, then there exists a solution $\left(u_{1}, u_{2}\right)$ of (1.1) such that $\left(u_{1}, u_{2}\right) \in\left[\underline{u}_{1}, \bar{u}_{1}\right] \times\left[\underline{u}_{2}, \bar{u}_{2}\right]$.

Remark 3.4. Observe that if $M_{i}$ is increasing, then it verifies $(N)$.

\section{Applications}

\subsection{Non-local Lotka-Volterra models}

Consider the classical diffusive Lotka-Volterra model with non-local interaction

$$
\begin{cases}-\Delta u_{1}=u_{1}\left(\lambda-u_{1}-b \int_{\Omega} u_{2}\right) & \text { in } \Omega \\ -\Delta u_{2}=u_{2}\left(\mu-u_{2}-c \int_{\Omega} u_{1}\right) & \text { in } \Omega \\ u_{1}=u_{2}=0 & \text { on } \partial \Omega\end{cases}
$$

where $\lambda, \mu \in \mathbb{R}$ and $b, c \in \mathbb{R}$. Here, $u_{1}$ and $u_{2}$ denote two species inhabiting in $\Omega$, the habitat, which is surrounded by inhospitable areas. Here, $\lambda$ and $\mu$ represent the intrinsic growth rates of each species, and $b, c$ the interaction rates between the species: if both $b$ and $c$ are positive numbers the species compete, if both are negative they cooperate and finally in the case $b>0$ and $c<0, u_{1}$ denotes the prey and $u_{2}$ the predator. The main 
novelty in (4.1) is that this interaction is non-local, that is, the interaction between both species at the point $x \in \Omega$ not only depends on the value at $x$ but the value to the entire domain $\Omega$, see [7].

In order to enunciate the main result, we need introduce some notation. Denote by $\varphi>0$ the eigenfunction associated to $\lambda_{1}$, the principal eigenvalue of the $-\Delta$ under Dirichlet boundary conditions, such that $\|\varphi\|_{\infty}=1$. It is well-known that the classical logistic equation

$$
\begin{cases}-\Delta w=w(\gamma-w) & \text { in } \Omega, \\ w=0 & \text { on } \partial \Omega,\end{cases}
$$

possesses a unique positive solution if and only if $\gamma>\lambda_{1}$. In such case, the positive solution is unique. We denote it by $\theta_{\gamma}$. We prolong the definition of $\theta_{\gamma} \equiv 0$ when $\gamma \leq \lambda_{1}$. It is well-known that $\gamma \mapsto \theta_{\gamma}$ is increasing in $\gamma$ and that $\theta_{\gamma} \leq \gamma$.

Theorem 4.1. 1. Assume that $b, c>0$. Then, (4.1) possesses at least a positive solution if

$$
\lambda-b \int_{\Omega} \theta_{\mu}>\lambda_{1} \quad \text { and } \quad \mu-c \int_{\Omega} \theta_{\lambda}>\lambda_{1}
$$

2. Assume that $b, c<0$ and $b c|\Omega|^{2}<1$. Then, (4.1) possesses at least a positive solution if $(\lambda, \mu)$ verifies condition (4.3).

3. Assume $b>0, c<0$ and

$$
\lambda-b|\Omega|\left(\mu+c \int_{\Omega} \theta_{\lambda}\right)>\lambda_{1} \quad \text { and } \quad \mu>\lambda_{1} .
$$

Proof. 1. We can take as pair of sub-supersolution

$$
\left(\underline{u}_{1}, \bar{u}_{1}\right)=\left(\theta_{\lambda-b \int_{\Omega} \theta_{\mu}}, \theta_{\lambda}\right), \quad\left(\underline{u}_{2}, \bar{u}_{2}\right)=\left(\theta_{\mu-c \int_{\Omega} \theta_{\lambda}}, \theta_{\mu}\right) .
$$

First, observe that $\underline{u}_{1} \leq \bar{u}_{1}$ and $\underline{u}_{2} \leq \bar{u}_{2}$ in $\Omega$. Now, we have to verify four inequalities. Let us only check two of them:

$$
-\Delta \underline{u}_{1} \leq \underline{u}_{1}\left(\lambda-\underline{u}_{1}-b \int_{\Omega} \bar{u}_{2}\right), \quad-\Delta \bar{u}_{1} \geq \bar{u}_{1}\left(\lambda-\bar{u}_{1}-b \int_{\Omega} \underline{u}_{2}\right) .
$$

Observe that

$$
-\Delta \underline{u}_{1}=-\Delta \theta_{\lambda-b \int_{\Omega} \theta_{\mu}}=\theta_{\lambda-b \int_{\Omega} \theta_{\mu}}\left(\lambda-b \int_{\Omega} \theta_{\mu}-\theta_{\lambda-b \int_{\Omega} \theta_{\mu}}\right)=\underline{u}_{1}\left(\lambda-\underline{u}_{1}-b \int_{\Omega} \bar{u}_{2}\right) .
$$

On the other hand,

$$
-\Delta \bar{u}_{1}=-\Delta \theta_{\lambda}=\theta_{\lambda}\left(\lambda-\theta_{\lambda}\right) \geq \theta_{\lambda}\left(\lambda-\theta_{\lambda}-b \int_{\Omega} \underline{u}_{2}\right)=\bar{u}_{1}\left(\lambda-\bar{u}_{1}-b \int_{\Omega} \underline{u}_{2}\right) .
$$

This completes the first paragraph.

2. In this case, take

$$
\left(\underline{u}_{1}, \bar{u}_{1}\right)=\left(\theta_{\lambda-b \int_{\Omega} \theta_{\mu}}, M\right), \quad\left(\underline{u}_{2}, \bar{u}_{2}\right)=\left(\theta_{\mu-c \int_{\Omega} \theta_{\lambda}}, N\right),
$$


where $M, N$ are positive constants verifying

$$
M \geq \lambda-b N|\Omega| \quad \text { and } \quad N \geq \mu-c M|\Omega|
$$

which exist because $b c|\Omega|^{2}<1$.

We prove now that they are sub-supersolutions. Again we only show two inequalities:

$$
-\Delta \underline{u}_{1} \leq \underline{u}_{1}\left(\lambda-\underline{u}_{1}-b \int_{\Omega} \underline{u}_{2}\right), \quad-\Delta \bar{u}_{1} \geq \bar{u}_{1}\left(\lambda-\bar{u}_{1}-b \int_{\Omega} \bar{u}_{2}\right) .
$$

The first inequality is equivalent to

$$
\theta_{\mu} \leq \theta_{\mu-c \int_{\Omega} \theta_{\lambda}}
$$

and the second one to

$$
0 \geq \lambda-M-b N|\Omega| .
$$

Taking $M$ and $N$ large we get both inequalities and $\underline{u}_{1} \leq \bar{u}_{1}$ and $\underline{u}_{2} \leq \bar{u}_{2}$.

3. Take in this case

$$
\left(\underline{u}_{1}, \bar{u}_{1}\right)=\left(\varepsilon \varphi, \theta_{\lambda}\right), \quad\left(\underline{u}_{2}, \bar{u}_{2}\right)=\left(\theta_{\mu}, N\right),
$$

with $\varepsilon, N>0$ to choose. Observe that $N$ has to verify that $N \geq \mu-c \int_{\Omega} \bar{u}_{1}$, and so, we can take

$$
N=\mu-c \int_{\Omega} \theta_{\lambda}
$$

It is clear that $\bar{u}_{1}$ and $\underline{u}_{2}$ verify the inequalities. Finally, we consider $\underline{u}_{1}$. It has to verify that

$$
\lambda_{1} \leq \lambda-\varepsilon \varphi-b N|\Omega|,
$$

so, if $\lambda-b N|\Omega|>\lambda_{1}$ we can take $\varepsilon$ small enough that the above inequality holds and $\underline{u}_{1} \leq \bar{u}_{1}$. Finally, observe that since $\theta_{\mu} \leq \mu<N$ we get that $\underline{u}_{2} \leq \bar{u}_{2}$.

\subsection{Kirchhoff systems}

Along this section, we assume that $M_{i}$ verifies $(M)$ and $(N)$. We present different applications of Theorem 3.3. First, we study a system with concave nonlinearities

$$
\begin{cases}-M_{1}\left(\left\|u_{1}\right\|^{2}\right) \Delta u_{1}=\lambda u_{1}^{q_{1}}+u_{2}^{q_{2}} & \text { in } \Omega \\ -M_{2}\left(\left\|u_{2}\right\|^{2}\right) \Delta u_{2}=\mu u_{2}^{p_{2}}+u_{1}^{p_{1}} & \text { in } \Omega \\ u_{1}=u_{2}=0 & \text { on } \partial \Omega\end{cases}
$$

where $\lambda, \mu \in \mathbb{R}$ and $0<q_{i}, p_{i}<1$.

Theorem 4.2. Assume that $\lambda, \mu>0$. Then, there exists a positive solution of (4.5).

Proof. We are going to build again a pair of sub-supersolution. Denote also by $e$ the unique positive solution of

$$
\begin{cases}-\Delta e=1 & \text { in } \Omega \\ e=0 & \text { on } \partial \Omega .\end{cases}
$$


We show that $\left(\underline{u}_{1}, \bar{u}_{1}\right)=\left(\varepsilon_{1} \varphi, K_{1} e\right)$ and $\left(\underline{u}_{2}, \bar{u}_{2}\right)=\left(\varepsilon_{2} \varphi, K_{2} e\right)$ is a pair of sub-supersolution of (4.5) taking the positive constants $\varepsilon_{1}, \varepsilon_{2}, K_{1}$ and $K_{2}$ in an appropriate way. We start with $\bar{u}_{1}$. We need to verify that

$$
-\mathcal{R}_{1}(u, v) \Delta \bar{u}_{1} \geq \lambda \bar{u}_{1}^{q_{1}}+\bar{u}_{2}^{q_{2}}, \quad \forall(u, v) \in\left[\underline{u}_{1}, \bar{u}_{1}\right] \times\left[\underline{u}_{2}, \bar{u}_{2}\right] .
$$

Using (M), it suffices to show that

$$
K_{1} m_{0} \geq \lambda K_{1}^{q_{1}}\|e\|_{\infty}^{q_{1}}+K_{2}^{q_{2}}\|e\|_{\infty}^{q_{2}} .
$$

Similarly for $\bar{u}_{2}$,

$$
K_{2} m_{0} \geq \mu K_{2}^{p_{2}}\|e\|_{\infty}^{p_{2}}+K_{1}^{p_{1}}\|e\|_{\infty}^{p_{1}} .
$$

Fix, $K_{1}$ and $K_{2}$ verifying above inequalities. Now, we study $\underline{u}_{1}$ and $\underline{u}_{2}$. They have to verify

$$
\begin{array}{ll}
\mathcal{R}_{1}(u, v) \lambda_{1} \varepsilon_{1} \varphi \leq \lambda\left(\varepsilon_{1} \varphi\right)^{q_{1}}+\left(\varepsilon_{2} \varphi\right)^{q_{2}}, & \forall(u, v) \in\left[\underline{u}_{1}, \bar{u}_{1}\right] \times\left[\underline{u}_{2}, \bar{u}_{2}\right], \\
\mathcal{R}_{2}(u, v) \lambda_{1} \varepsilon_{2} \varphi \leq \mu\left(\varepsilon_{2} \varphi\right)^{p_{2}}+\left(\varepsilon_{1} \varphi\right)^{p_{1}}, & \forall(u, v) \in\left[\underline{u}_{1}, \bar{u}_{1}\right] \times\left[\underline{u}_{2}, \bar{u}_{2}\right] .
\end{array}
$$

Since $\mathcal{R}_{i}$ is bounded in $\left[0, \bar{u}_{1}\right] \times\left[0, \bar{u}_{2}\right]$, it is clear that we can take $\varepsilon_{1}$ and $\varepsilon_{2}$ small enough, and we conclude the result.

Finally, we consider the competition Kirchhoff model with local nonlinearities

$$
\begin{cases}-M_{1}\left(\left\|u_{1}\right\|^{2}\right) \Delta u_{1}=u_{1}\left(\lambda-u_{1}-b u_{2}\right) & \text { in } \Omega, \\ -M_{2}\left(\left\|u_{2}\right\|^{2}\right) \Delta u_{2}=u_{2}\left(\mu-u_{2}-c u_{1}\right) & \text { in } \Omega, \\ u_{1}=u_{2}=0 & \text { on } \partial \Omega\end{cases}
$$

where $\lambda, \mu \in \mathbb{R}$ and $0<b, c$. The meaning of the parameters were given at the beginning of this Section.

Theorem 4.3. Assume that there exist positive constants $m_{i}^{\infty}, i=1,2$, such that $M_{i} \leq$ $m_{i}^{\infty}$, and

$$
\lambda>b \mu+\lambda_{1} m_{1}^{\infty} \text { and } \quad \mu>c \lambda+\lambda_{1} m_{2}^{\infty} .
$$

Then, there exists a positive solution of (4.7).

Proof. We show that

$$
\left(\underline{u}_{1}, \bar{u}_{1}\right)=\left(\varepsilon_{1} \varphi, M_{1}\right) \quad \text { and } \quad\left(\underline{u}_{2}, \bar{u}_{2}\right)=\left(\varepsilon_{2} \varphi, M_{2}\right)
$$

is a pair of sub-supersolution of (4.7) taking positive constants $\varepsilon_{1}, \varepsilon_{2}$ small $M_{1}=\lambda$, $M_{2}=\mu$. Indeed, $\bar{u}_{1}$ is supersolution if

$$
0 \geq \lambda-M_{1}-b \varepsilon_{2} \varphi
$$

which is true for $M_{1}=\lambda$.

Consider now $\underline{u}_{1}$. The function $\underline{u}_{1}=\varepsilon_{1} \varphi$ is subsolution provided of

$$
\mathcal{R}_{1}(u, v) \lambda_{1} \varepsilon_{1} \varphi \leq\left(\varepsilon_{1} \varphi\right)\left(\lambda-\varepsilon_{1} \varphi-b \mu\right), \quad \forall(u, v) \in\left[\underline{u}_{1}, \bar{u}_{1}\right] \times\left[\underline{u}_{2}, \bar{u}_{2}\right],
$$

for which it suffices $\lambda>b \mu+\lambda_{1} m_{1}^{\infty}$. Analogously for $\underline{u}_{2}$ and $\bar{u}_{2}$.

Acknowledgements. The authors have been partially supported for the following projects: CNPQ-Proc. 400426/2013-7 (GF and AS), CNPQ-Proc. 301242/2011-9 (GF) and Ministerio de Economía y Competitividad under grant MTM2012-31304 (AS). 


\section{References}

[1] N. T. Chung, An existence result for a class of Kirchhoff type systems via sub and supersolutions method, Appl. Math. Lett. 35 (2014), 95-101.

[2] N. T. Chung and G. A. Afrouzi, Existence of positive solutions for a class of nonlocal elliptic systems with multiple parameter, (2014) to appear in Matematicki Vesnik.

[3] F. J. S. A. Corrêa and R. G. Nascimento, On a nonlocal elliptic system of p-Kirchhofftype under Neumann boundary condition, Math. Comput. Modelling 49 (2009), no. 3-4, 598-604.

[4] G. M. Figueiredo, On a nonlocal system with critical growth: Recent trends on Nonlinear Elliptic System, edited by N. Zographopoulos, International Scientific press, 2012, Greece.

[5] G. M. Figueiredo and A. Suárez, Some remarks on the comparison principle in Kirchhoff equations, submitted.

[6] S. Khademloo, E. Valipour and A. Babakhani, Multiplicity of positive solutions for a second order elliptic system of Kirchhoff type, preprint.

[7] H. Leman, S. Méléard and S. Mirrahimi, Influence of a spatial structure on the long time behavior of a competitive Lotka-Volterra type system, arXiv:1401.1182 (2014).

[8] Q. Li and Z. Yang, Existence of positive solutions for a quasilinear elliptic systems of p-Kirchhoff type, Differ. Equ. Appl. 6 (2014), no. 1, 73-80.

[9] C. V. Pao, "Nonlinear Parabolic and Elliptic Equations", Plenum Press, New York, 1992.

[10] Z. Zhang and Y. Suny, Existence and multiplicity of solutions for nonlocal systems with Kirchhoff type, arXiv:1410.6225v1. 